\title{
Theory of non-Markovian decay of a cascade atom in high-Q cavities and photonic band-gap materials
}

\author{
B.M. Garraway \\ Department of Physics and Astronomy, \\ University of Sussex, Falmer, Brighton, BN1 9QH, United Kingdom \\ B.J. Dalton \\ ARC Centre of Excellence for Quantum-Atom Optics, \\ and Centre for Atom Optics and Ultrafast Spectroscopy, \\ Swinburne University of Technology, \\ Melbourne, Victoria 3122, Australia
}

(Dated: October 8, 2018)

\begin{abstract}
The dynamics of a three-level atom in a cascade configuration with both transitions coupled to a single structured reservoir of quantized field modes is treated using Laplace transform methods applied to the coupled amplitude equations. Results are also obtained from master equations by two different approaches, that is, involving either pseudomodes or quasimodes. Two different types of reservoir are considered, namely a high-Q cavity and a photonic band-gap system, in which the respective reservoir structure functions involve Lorentzians. Non-resonant transitions are included in the model. In all cases non-Markovian behaviour for the atomic system can be found, such as oscillatory decay for the high-Q cavity case and population trapping for the photonic band-gap case. In the master equation approaches, the atomic system is augmented by a small number of pseudomodes or quasimodes, which in the quasimode approach themselves undergo Markovian relaxation into a flat reservoir of continuum quasimodes. Results from these methods are found to be identical to those from the Laplace transform method including two-photon excitation of the reservoir with both emitting sequences. This shows that complicated non-Markovian decays of an atomic system into structured EM field reservoirs can be described by Markovian models for the atomic system coupled to a small number of pseudomodes or quasimodes.

PACS numbers: 42.50.Ct, 03.65.Yz
\end{abstract}




\section{INTRODUCTION}

The study of open quantum systems, in which the interaction of the quantum system with the environment is taken into account, is of fundamental importance in several areas of physics. One such area is quantum optics [1], where the quantum system may be an atom (or an atom combined with a single mode laser field) and the environment with which the atom interacts may be a continuum of quantised modes of the electromagnetic field (or a set of vibrational modes in a solid). Another area is quantum measurement theory [2, 3], where the environment includes the macroscopic measuring apparatus, whose states "record" the results of measurements on the quantum system itself. Explaining the emergence of the classical world [3, 4] is related to the way the presence of the environment destroys coherence between certain states of macroscopic systems, resulting in only the "pointer states" remaining stable - any quantum superpositions of pointer states are rapidly converted into mixed states. A third area is that of degenerate quantum gases [5], where the quantum system is an atomic Bose-Einstein condensate or atomic Fermi gas, and the environment may consist of those atoms in thermally excited states, as distinct from being in the macroscopically occupied condensate state or in states within the Fermi surface. As in some of these examples, the quantum system itself may be microscopic or macroscopic.

In many cases the interaction between the quantum system and the environment (also referred to as the bath or reservoir) involves coupling constants and reservoir mode densities that have a slowly varying frequency dependence. In such cases the reservoir structure function (which is the product of the mode density with the square of the coupling constant) is also slowly varying, resulting in the reservoir correlation time (which is the inverse of the bandwidth for the reservoir structure function) being very short compared to the time scales for dynamic evolution of the quantum system. This situation enables the dynamical behaviour of the quantum system and its interaction with the environment to be described by Markovian master equations (see e.g. 1] ). A large literature exists where the dynamical behaviour of quantum systems coupled to the environment has been successfully explained via Markovian master equations and related methods, and indeed much of quantum optics (see [1, 6]) falls into this category.

In recent years however, there has been an interest in open quantum systems where the conditions required for Markovian behaviour do not necessarily apply. Cases include atom 
lasers [7], quantum Brownian motion [8, 9], systems with conditioned evolution (such as associated with photodetection) [10], decoherence in large scale quantum computers [11, 12, 13] and in macroscopic systems generally [14, 15, 16]. A further non-Markovian situation occurs for atomic systems coupled to structured reservoirs of electromagnetic (EM) field modes, where either the coupling constants or the mode density (or both) change rapidly with frequency. This situation can occur for atoms in high-Q cavities or in photonic bandgap (PBG) systems, and a general review of such situations is given by Lambropoulos et al. [17].

A number of methods for treating non-Markovian problems have been formulated. These include: non-Markovian master equations [18, 19, 20, 21]; the time-convolutionless projection operator master equation [8, 9, 22]; Heisenberg equations of motion 23]; stochastic wave function methods for non-Markovian processes [8, 10, 24, 25, 26, 27, 28, 29, 30]; methods based on the essential states approximation or resolvent operators [17, 31, 32, 33]; the pseudomode approach [34, 35]; Fano diagonalisation [36, 37]; and various short time scale methods [14, 15, 38, 39, 40, 41, 42]. The last four approaches are less powerful in the formal sense, but often simpler to apply and interpret.

In recent work $([43,44$, 45, 46]) we have studied non-Markovian processes for atomic systems coupled to a structured reservoirs of EM field modes, with applications to highQ cavities and PBG systems [17]. Our approach has been based on the essential states approximation, Fano diagonalisation and pseudomodes. In Ref.[46] the case of a three level cascade (or ladder) system coupled to a structured reservoir of EM modes was treated using the essential states approach, following a method similar to [33]. The general equations for the coupled amplitudes and their Laplace transforms were obtained, and the resulting integral equation solved via numerical methods, based on discretising frequency space to give a matrix equation that is equivalent to the original integral equation and also utilising analytical continuation in the complex $s$ plane. The decay of the initially excited upper state was found as a function of time. Our application in [46] was restricted to the case where the structured reservoir was due to a high-Q cavity. In the present paper, we apply the same method also to the case where the structured reservoir is due to a model photonic band-gap. In addition some further high-Q cavity cases are examined, where the cavity resonance is detuned from the atomic transition frequency. Other non-Markovian studies of the cascade system in a PBG system have been carried out in Refs. [47, 48]. In these papers 
a discontinuous mode density is used, whereas we represent the PBG by a difference of two Lorentzian functions. Quantitative comparisons between their results and ours are therefore not possible, only general features such as population trapping effects are in common. For completeness and to set out the notation, we include here in Section II the key integral equations for the cascade system decay, based on the essential states approach, that were derived in [46]. Results for both the high-Q cavity and PBG cases are presented in Section III. The dynamics is also treated via master equations by two different approaches, that is, involving either pseudomodes or quasimodes. In the master equation approach, the atomic system is augmented by a small number of pseudomodes or quasimodes, which in the quasimode approach themselves undergo Markovian relaxation into a flat reservoir of continuum quasimodes. The master equation theory is presented in Section IV] with the pseudomode approach [34, 35] being described in Subsection IVA and a treatment based on quasimodes [43, 44] given in Subsection IVB. Results from the master equation method are compared to those from the Laplace transform method. A summary of the paper is set out in Section $\nabla$

\section{ESSENTIAL STATES THEORY}

\section{A. Model System}

The system consists of a three level cascade atom, shown in figure 11 coupled to a bath (or reservoir) of bosonic modes. The bath modes would usually be associated with the quantum EM field, but other bosonic baths (such as lattice vibrations in a solid) might realise a similar model. For convenience, we will refer to the bath quanta as photons, but other quanta could be involved (such as phonons in the lattice vibration case).

The atomic states are illustrated in figure 1] the energy difference between states 1 and 0 is $\hbar \omega_{1}$, and between 2 and 1 the difference is $\hbar \omega_{2}$. For later use we also define a frequency offset $\bar{\delta}$, such that

$$
\bar{\delta}=\left(\omega_{1}-\omega_{2}\right) / 2
$$

The offset determines the energy difference between the two transitions. If the offset is large, we would expect the system to behave as though there are two independent reservoirs. However, for relatively small offsets a photon may well be emitted from one transition, and 


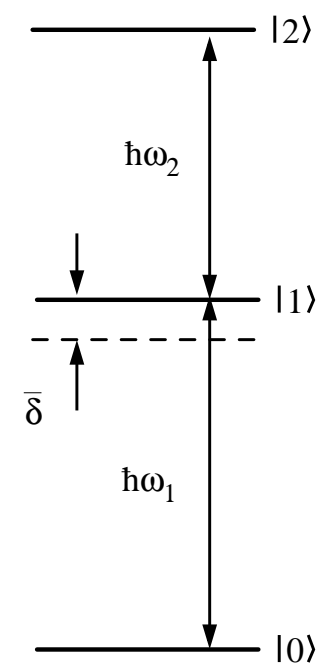

FIG. 1: Energy levels and energy differences in the three level system. The off-set $\bar{\delta}$ represents the difference between the energy of level 1 and the midpoint between levels 0 and 2 .

later re-absorbed on the other transition. The bath modes have frequencies $\omega_{\lambda}$, raising and lowering operators $\hat{a}_{\lambda}^{\dagger}$ and $\hat{a}_{\lambda}$ and the density of modes is $\rho_{\lambda}$. Frequency dependent coupling constants $g_{\lambda 1}$ and $g_{\lambda 2}$ specify the coupling between atomic transitions $0-1$ and $1-2$ and the reservoir.

The Hamiltonian for the system in the rotating wave approximation is given by:

$$
\begin{aligned}
\hat{H}= & \hbar\left[\omega_{1}|1\rangle\left\langle 1\left|+\left(\omega_{1}+\omega_{2}\right)\right| 2\right\rangle\langle 2|+\sum_{\lambda} \omega_{\lambda} \hat{a}_{\lambda}^{\dagger} \hat{a}_{\lambda}\right. \\
& \left.+\sum_{\lambda}\left[g_{\lambda 1}\left(\hat{a}_{\lambda}^{\dagger}|0\rangle\left\langle 1\left|+\hat{a}_{\lambda}\right| 1\right\rangle\langle 0|\right)+g_{\lambda 2}\left(\hat{a}_{\lambda}^{\dagger}|1\rangle\left\langle 2\left|+\hat{a}_{\lambda}\right| 2\right\rangle\langle 1|\right)\right]\right] .
\end{aligned}
$$

Initially the atom is assumed to be in the upper state $|2\rangle$ and the bath modes are empty of photons, so the initial state vector is

$$
|\Psi(0)\rangle=|2\rangle\left|0_{\lambda}\right\rangle
$$

The atom plus bath system evolves according to the Schrödinger equation. The initial state assumed together with the rotating wave approximation allows for the atomic state $|1\rangle$ to be coupled to one photon bath states and atomic state $|0\rangle$ to be coupled to bath states with two photons - the latter may be associated with the same mode or two different modes. 


\section{B. Coupled Amplitude Equations}

The time evolving state vector is written in the form:

$$
\begin{aligned}
|\Psi(t)\rangle= & c_{2} e^{-i\left(\omega_{1}+\omega_{2}\right) t}|2\rangle\left|0_{\lambda}\right\rangle+\sum_{\lambda} c_{1 \lambda} e^{-i\left(\omega_{1}+\omega_{\lambda}\right) t}|1\rangle\left|1_{\lambda}\right\rangle \\
& +\sum_{\lambda} c_{0 \lambda \lambda} e^{-2 i \omega_{\lambda} t}|0\rangle\left|2_{\lambda}\right\rangle+\sum_{\lambda, \mu, \lambda<\mu} c_{0 \lambda \mu} e^{-i\left(\omega_{\lambda}+\omega_{\mu}\right) t}|0\rangle\left|1_{\lambda} 1_{\mu}\right\rangle,
\end{aligned}
$$

where $c_{2}, c_{1 \lambda}, c_{0 \lambda \lambda}$ and $c_{0 \lambda \mu}$ are interaction picture amplitudes for the various states. A straight-forward substitution into the Schrödinger equation leads to a set of coupled equations for these amplitudes.

The overall aim is to find the evolution of the atomic initial state, e.g. $c_{2}(t)$. We first obtain a set of coupled equations for the Laplace transforms of the amplitude equations, and then apply the approach of [33] to make a convenient change of the amplitudes by incorporating the coupling constants via $\bar{c}_{2}=\bar{b}_{2}, \bar{c}_{1 \lambda}=g_{\lambda 2} \bar{b}_{1 \lambda}, \bar{c}_{0 \lambda \mu}=g_{\lambda 2} g_{\mu 1} \bar{b}_{0 \lambda \mu} \quad(\lambda<\mu)$ and $\bar{c}_{0 \lambda \lambda}=g_{\lambda 2} g_{\lambda 1} \bar{b}_{0 \lambda \lambda}$. Laplace transforms are denoted by a bar, and associated with the complex variable $s$. The coupled equations obtained are [46]:

$$
\begin{aligned}
s \bar{b}_{2}(s)-1= & -i \sum_{\lambda} g_{\lambda 2}^{2} \bar{b}_{1 \lambda}\left(s+i\left(\omega_{\lambda}-\omega_{2}\right)\right) \\
s \bar{b}_{1 \lambda}(s)= & -i \sum_{\mu,(\mu>\lambda)} g_{\mu 1}^{2} \bar{b}_{0 \lambda \mu}\left(s+i\left(\omega_{\mu}-\omega_{1}\right)\right) \\
& -i \sum_{\mu,(\mu<\lambda)} g_{\mu 1}^{2} \alpha_{\lambda \mu} \bar{b}_{0 \mu \lambda}\left(s+i\left(\omega_{\mu}-\omega_{1}\right)\right) \\
& -i g_{\lambda 1}^{2} \sqrt{2} \bar{b}_{0 \lambda \lambda}\left(s+i\left(\omega_{\lambda}-\omega_{1}\right)\right)-i \bar{b}_{2}\left(s+i\left(\omega_{2}-\omega_{\lambda}\right)\right) \\
s \bar{b}_{0 \lambda \lambda}(s)= & -i \sqrt{2} \bar{b}_{1 \lambda}\left(s+i\left(\omega_{1}-\omega_{\lambda}\right)\right) \\
s \bar{b}_{0 \lambda \mu}(s)= & -i \bar{b}_{1 \lambda}\left(s+i\left(\omega_{1}-\omega_{\mu}\right)\right)-i \alpha_{\lambda \mu} \bar{b}_{1 \mu}\left(s+i\left(\omega_{1}-\omega_{\lambda}\right)\right) .
\end{aligned}
$$

These involve a frequency independent parameter:

$$
\alpha_{\lambda \mu}=\frac{g_{\lambda 1} g_{\mu 2}}{g_{\lambda 2} g_{\mu 1}}
$$

It is not possible to obtain explicit solutions for the new Laplace transform amplitudes. However, we can proceed further by first eliminating $\bar{b}_{0 \lambda \mu}, \bar{b}_{0 \lambda \lambda}$ in the above equations, leading to a set of coupled equations for the remaining amplitudes $\bar{b}_{2}(s)$ and $\bar{b}_{1 \mu}(s)$. 


\section{Integral Equation}

We then eliminate $\bar{b}_{2}(s)$ in favour of $\bar{b}_{1 \mu}(s)$ (though not the reverse) giving a set of equations (see [46]) for the remaining amplitude $\bar{b}_{1 \mu}(s)$ :

$$
\begin{aligned}
-i / s= & \left(s+i\left(\omega_{\lambda}-\omega_{2}\right)+\sum_{\eta} \frac{g_{\eta 1}^{2}}{s+i\left(\omega_{\lambda}+\omega_{\eta}-\omega_{1}-\omega_{2}\right)}\right) \bar{b}_{1 \lambda}\left(s+i\left(\omega_{\lambda}-\omega_{2}\right)\right. \\
& +\sum_{\mu}\left(g_{\mu 1}^{2} \frac{g_{\lambda 1} g_{\mu 2}}{g_{\lambda 2} g_{\mu 1}} \frac{1}{s+i\left(\omega_{\lambda}+\omega_{\mu}-\omega_{1}-\omega_{2}\right)}+\frac{g_{\mu 2}^{2}}{s}\right) \bar{b}_{1 \mu}\left(s+i\left(\omega_{\mu}-\omega_{2}\right)\right) .
\end{aligned}
$$

The above equation is for the amplitude $\bar{b}_{1 \lambda}(s)$ - it is of course coupled to all similar amplitudes $\bar{b}_{1 \mu}(s)$.

By converting the sums to integrals, i.e. $\sum_{\mu} \longrightarrow \int d \omega_{\mu} \rho\left(\omega_{\mu}\right)$, we obtain a Fredholm integral equation of the second kind [46]

$$
\bar{f}\left(\omega_{\lambda}\right)+\int d \omega_{\mu} K\left(\omega_{\lambda}, \omega_{\mu}\right) \bar{f}\left(\omega_{\mu}\right)=d\left(\omega_{\lambda}\right)
$$

with

$$
\begin{aligned}
\bar{f}\left(\omega_{\lambda}\right) & =\bar{b}_{1 \lambda}\left(s+i\left(\omega_{\lambda}-\omega_{2}\right)\right) \\
K\left(\omega_{\lambda}, \omega_{\mu}\right) & =B\left(\omega_{\lambda}, \omega_{\mu}\right) / A\left(\omega_{\lambda}\right) \\
d\left(\omega_{\lambda}\right) & =C / A\left(\omega_{\lambda}\right) \\
A\left(\omega_{\lambda}\right) & =s+i\left(\omega_{\lambda}-\omega_{2}\right)+\int d \omega_{\eta} \rho\left(\omega_{\eta}\right) \frac{g_{\eta 1}^{2}}{s+i\left(\omega_{\lambda}+\omega_{\eta}-\omega_{1}-\omega_{2}\right)} \\
B\left(\omega_{\lambda}, \omega_{\mu}\right) & =\rho\left(\omega_{\mu}\right)\left(g_{\mu 1}^{2} \frac{g_{\lambda 1} g_{\mu 2}}{g_{\lambda 2} g_{\mu 1}} \frac{1}{s+i\left(\omega_{\lambda}+\omega_{\mu}-\omega_{1}-\omega_{2}\right)}+\frac{g_{\mu 2}^{2}}{s}\right) \\
C & =-i / s
\end{aligned}
$$

The quantities $\bar{f}\left(\omega_{\lambda}\right), K\left(\omega_{\lambda}, \omega_{\mu}\right), A\left(\omega_{\lambda}\right), B\left(\omega_{\lambda}, \omega_{\mu}\right), C$ and $d\left(\omega_{\lambda}\right)$ are all functions of the Laplace variable $s$. The quantity $K$ performs the role of the kernel for the integral equation for the quantity $\bar{f}$, and the quantity $d$ makes the integral equation inhomogeneous.

As pointed out in [46], the integral equations for $\bar{b}_{1 \lambda}(s)$ and the other related equations for $\bar{b}_{2}(s)$ and $\bar{b}_{0 \lambda \mu}(s)$ only involve the reservoir quantities $g_{\lambda 1}, g_{\lambda 2}, \rho_{\lambda}$ in terms of reservoir structure functions

$$
\begin{aligned}
& R_{1}\left(\omega_{\lambda}\right)=\rho\left(\omega_{\lambda}\right) g_{\lambda 1}^{2} \\
& R_{2}\left(\omega_{\lambda}\right)=\rho\left(\omega_{\lambda}\right) g_{\lambda 2}^{2}
\end{aligned}
$$


Since the atomic density operator

$$
\widehat{\rho}_{A}=\operatorname{Tr}_{F}|\Psi\rangle\langle\Psi|
$$

also only involves reservoir structure functions and the reduced amplitudes $b_{2}(t), b_{1 \lambda}(t)$ and $b_{0 \lambda \mu}(t)$, an important result follows that the overall dynamics is entirely determined by the reservoir structure functions.

The integral equation (11) can be solved in different ways, such as: (a) numerical methods in which the integral equation is converted to a matrix equation (see next sections); (b) expansions using bi-orthogonal eigenfunctions (see Ref. [46]); (c) expansions such as the Fredholm expansion (see textbooks on integral equations).

\section{Numerical Solution of Integral Equation}

By discretising the mode frequencies $\omega_{\lambda}$ the integral equation (11) can be converted to a matrix equation (the rows and columns are specified by the discrete frequencies and the integral over $\omega_{\mu}$ is approximated by a discrete sum) of the form

$$
(\mathbf{K}+\mathbf{I}) \overline{\mathbf{f}}=\mathbf{d}
$$

which we then can solve for $\overline{\mathbf{f}}$.

However, the obvious simple solution of the form $\overline{\mathbf{f}}=(\mathbf{K}+\mathbf{I})^{-1} \mathbf{d}$ is not correct. To get the time evolution of $b_{2}$ for example, we need both its real and imaginary parts, $b_{2 r}$ and $b_{2 i}$. This means we need the separate Laplace transforms $\bar{b}_{2 r}(s)$ and $\bar{b}_{2 i}(s)$ to invert, and this in turn requires us to separately obtain $\overline{f_{r}}(s)$ and $\overline{f_{i}}(s)$. Thus we need the separate real and imaginary parts of $\bar{f}$, both being functions of the complex Laplace variable $s$. These separate parts are obtained by analytic continuation of the real and imaginary parts of $K\left(\omega_{\lambda}, \omega_{\mu}\right)$,

$\bar{f}\left(\omega_{\lambda}\right)$ and $D\left(\omega_{\lambda}\right)$ on the real $s$-axis. In other words, we first break the equation $(\mathbf{K}+\mathbf{I}) \overline{\mathbf{f}}=\mathbf{d}$ into its real and imaginary parts on the basis of the variable $s$ being real, solve for $\overline{f_{r}}(s)$ and $\overline{f_{i}}(s)$ with real $s$, then obtain $\overline{f_{r}}(s)$ and $\overline{f_{i}}(s)$ for complex $s$ by analytic continuation.

In matrix form in terms of the real and imaginary parts, the integral equation (21) becomes

$$
\left(\begin{array}{c|c}
\mathbf{K}_{r}+\mathbf{I} & -\mathbf{K}_{i} \\
\hline \mathbf{K}_{i} & \mathbf{K}_{r}+\mathbf{I}
\end{array}\right)\left(\begin{array}{c}
\overline{\mathbf{f}_{r}} \\
\overline{\overline{\mathbf{f}_{i}}}
\end{array}\right)=\left(\begin{array}{c}
\mathbf{d}_{r} \\
\hline \mathbf{d}_{i}
\end{array}\right) .
$$


As emphasised above, the real, imaginary parts are denoted $\mathbf{K}_{r}, \mathbf{K}_{i}$ etc. and are identified for $s$ real. Analytic continuation enables solution for $\overline{\mathbf{f}_{r}}$ and $\overline{\mathbf{f}_{i}}$ which will apply for all $s$.

Solving (22) for $\overline{\mathbf{f}_{r}}$ and $\overline{\mathbf{f}_{i}}$ by matrix inversion, gives the LT amplitude $\bar{b}_{1 \lambda}\left(s+i\left(\omega_{\lambda}-\omega_{2}\right)\right)$. The coupled amplitude equations (5) give $\bar{b}_{2}(s)$. For the discretised frequency form, the real and imaginary parts can be written as the scalar products [46]

$$
\begin{aligned}
& \bar{b}_{2 r}(s)=\left(1+\mathbf{r} \cdot \overline{\mathbf{f}_{i}}\right) / s \\
& \bar{b}_{2 i}(s)=-\mathbf{r} \cdot \overline{\mathbf{f}_{r}} / s,
\end{aligned}
$$

where $\mathbf{r} \equiv\left\{\rho_{\lambda} g_{\lambda 2}^{2}\right\}$. The amplitude $b_{2}(t)$ then follows from a numerical inverse Laplace transform of both $\bar{b}_{2 r}(s)$ and $\bar{b}_{2 i}(s)$.

\section{APPLICATIONS}

\section{A. Lorentzian resonances}

Before looking at two special cases, we will first generate some results for a reservoir structure composed of a sum of Lorentzian resonances. The resonances need not be well separated from each other in frequency space, but must be well above zero frequency. The general form of structure function is then:

$$
\rho g_{1}^{2}(\omega)=\eta \rho g_{2}^{2}(\omega)=\sum_{\alpha} Z_{\alpha} \cdot \frac{\Gamma_{\alpha}}{2 \pi} \cdot \frac{1}{\left(\omega-\omega_{\alpha}^{c}\right)^{2}+\left(\Gamma_{\alpha} / 2\right)^{2}},
$$

where a simple scaling $\eta$ has been introduced between the strength of coupling for the upper and lower transitions, $g_{\lambda 1}^{2}=\eta g_{\lambda 2}^{2}$. In this situation $\alpha_{\lambda \mu}=1$, [see equation (91)]. The $\alpha$ th Lorentzian is weighted by $Z_{\alpha}$ (not necessarily positive) and has a resonance centred at the frequency $\omega_{\alpha}^{c}$ with width $\Gamma_{\alpha}$.

For these cases of reservoir structure function, the integral in equation (15) is straightforward and gives for the function $A$

$$
A\left(\omega_{\lambda}\right)=s+i\left(\omega_{\lambda}-\omega_{2}\right)+\sum_{\alpha} \frac{Z_{\alpha}}{s+\Gamma_{\alpha} / 2+i\left(\omega_{\lambda}+\omega_{\alpha}^{c}-\omega_{1}-\omega_{2}\right)} .
$$

In what follows we define the reservoir frequency and resonances relative to the average of the transition frequencies, i.e. we let

$$
\begin{aligned}
\Delta \omega & =\omega_{\lambda}-\left(\omega_{1}+\omega_{2}\right) / 2 \\
\Delta \omega^{\prime} & =\omega_{\mu}-\left(\omega_{1}+\omega_{2}\right) / 2
\end{aligned}
$$


and define

$$
\delta_{\alpha}=\omega_{\alpha}^{c}-\left(\omega_{1}+\omega_{2}\right) / 2
$$

The detuning $\delta_{\alpha}$ is then the offset of the $\alpha$ th Lorentzian from the average frequency such that if $\delta_{\alpha}= \pm \bar{\delta}$ the $\alpha$ th Lorentzian is resonant with $\omega_{1}$, or $\omega_{2}$, according to the sign of $\bar{\delta}$. Then, together with equation (II) for $\bar{\delta}$, we find in this notation that

$$
A(\Delta \omega)=s+i(\Delta \omega+\bar{\delta})+\sum_{\alpha} \frac{Z_{\alpha}}{s+\Gamma_{\alpha} / 2+i\left(\Delta \omega+\delta_{\alpha}\right)}
$$

and

$$
B\left(\Delta \omega, \Delta \omega^{\prime}\right)=\left[\frac{1}{\eta s}+\frac{1}{s+i\left(\Delta \omega+\Delta \omega^{\prime}\right)}\right] \sum_{\alpha} Z_{\alpha} \cdot \frac{\Gamma_{\alpha}}{2 \pi} \cdot \frac{1}{\left(\Delta \omega^{\prime}-\delta_{\alpha}\right)^{2}+\left(\Gamma_{\alpha} / 2\right)^{2}} .
$$

As before, the kernel is then $K\left(\Delta \omega, \Delta \omega^{\prime}\right)=B\left(\Delta \omega, \Delta \omega^{\prime}\right) / A(\Delta \omega)$.

\section{B. Case A: High-Q Cavity Model}

In this section we consider the special case of the three-level atom coupled to a single high-Q cavity mode. For simplicity, identical coupling constants will be assumed. The atomic transition frequencies may be unequal. Thus we have $g_{\lambda 1}=g_{\lambda 2}=g_{\lambda}$, and $\eta=1$ in equation (25). In this case the frequency dependence of the reservoir structure function will be due to resonant behaviour of the coupling constants, as a quasimode theory of such systems demonstrates [45]. Thus, parameterising the single weight $Z_{1}$ by $\Omega^{2}$ we have:

$$
\begin{aligned}
R_{1} & =R_{2}=R=\rho_{\lambda} g_{\lambda}^{2} \\
& =\frac{\Gamma \Omega^{2}}{2 \pi} \cdot \frac{1}{(\Delta \omega-\delta)^{2}+(\Gamma / 2)^{2}},
\end{aligned}
$$

where we denote the single detuning $\delta_{1}$ by $\delta$.

The kernel $K$ [see equation (13)] is given by:

$$
K\left(\Delta \omega, \Delta \omega^{\prime}\right)=\frac{\Gamma \Omega^{2}}{2 \pi} \frac{(s+i(\Delta \omega+\delta)+\Gamma / 2)\left(2 s+i\left(\Delta \omega+\Delta \omega^{\prime}\right)\right.}{s\left(\left(\Delta \omega^{\prime}-\delta\right)^{2}+(\Gamma / 2)^{2}\right)\left(s+i\left(\Delta \omega+\Delta \omega^{\prime}\right)\right) Q(\Delta \omega)},
$$

where $Q(\Delta \omega)=[s+i(\Delta \omega+\bar{\delta})][s+i(\Delta \omega+\delta)+\Gamma / 2]+\Omega^{2}$. The frequency integral in equation (13) is integrated over the range $\pm \infty$ for convenience. The matrices $\mathbf{K}_{r}, \mathbf{K}_{i}$ are determined from the kernel $K$ and the matrices $\mathbf{d}_{r}, \mathbf{d}_{i}$ from an analogous equation for $d$. The amplitude $b_{2}(t)$ is obtained by numerical integration. 


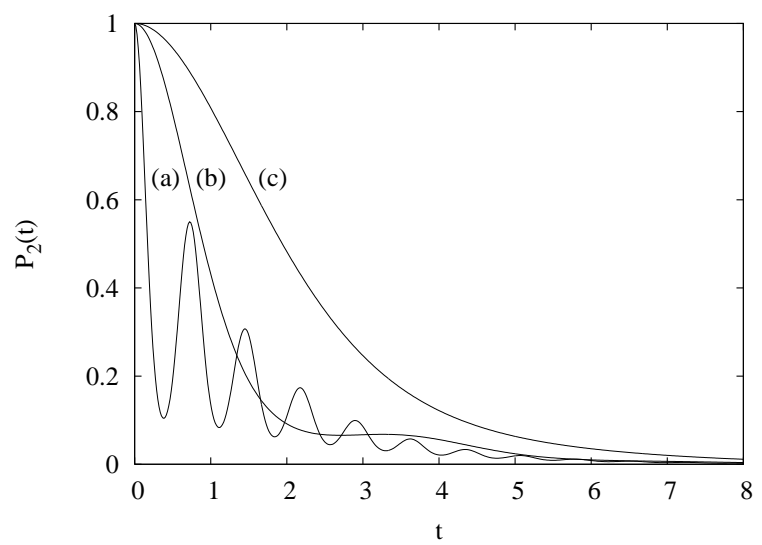

FIG. 2: High-Q cavity model. Time evolution of the probability of finding the system in state 2; $P(t)=\left|b_{2}(t)\right|^{2}$. The reservoir structure function is given by equation (32), with parameters $\Gamma=1$ and: (a) $\Omega=5.0$; (b) $\Omega=1.0$; and (c) $\Omega=0.5$, in scaled units. The grid size for the discretised kernel was $150 \times 150$ chosen with a range of \pm 30 for $\Delta \omega$ and $\Delta \omega^{\prime}$ in scaled units. The case of resonance is shown, where each transition is resonant with the reservoir structure: $\bar{\delta}=\delta=0$. Results were computed with the integral equation method of section IID.

We note that in the special case of a structure resonant with both the transitions, $\omega_{1}=$ $\omega_{2}=\omega_{0}=\omega_{1}^{c}$ and $\delta=\bar{\delta}=0$. The kernel then reduces to the case studied in [46], i.e.

$$
K\left(\Delta \omega, \Delta \omega^{\prime}\right)=\frac{\Gamma \Omega^{2}}{2 \pi} \frac{(s+i \Delta \omega++\Gamma / 2)\left(2 s+i\left(\Delta \omega+\Delta \omega^{\prime}\right)\right.}{s\left(\Delta \omega^{\prime 2}+(\Gamma / 2)^{2}\right)\left(s+i\left(\Delta \omega+\Delta \omega^{\prime}\right)\right) Q(\Delta \omega)},
$$

where now $Q(\Delta \omega)=(s+i \Delta \omega)(s+i \Delta \omega+\Gamma / 2)+\Omega^{2}$.

Typical results for the time-dependent upper state probability are shown in figure 2 , where the parameters used are $\Gamma=1$ and (a) $\Omega=5$ (b) $\Omega=1$ (c) $\Omega=0.5$ (effectively in scaled units). The damped oscillation in case (a) is typical of non-Markovian decay. In case (b) the oscillations weaken. Further reduction in coupling in case (c) removes the oscillations and the decay is closer to exponential.

The case just described applies when both transitions are coupled to a single reservoir. A different situation applies when the two transitions are coupled to two separate reservoirs, and the results for this case are presented in [46]. The equations are then simpler, and the integral equation for the amplitudes can be solved analytically. The decay of the excited state is now less oscillatory. Unlike the single reservoir case, the two photons emitted can now be distinguished.

The typical effects of detunings on the upper state probability are presented in figures 3 


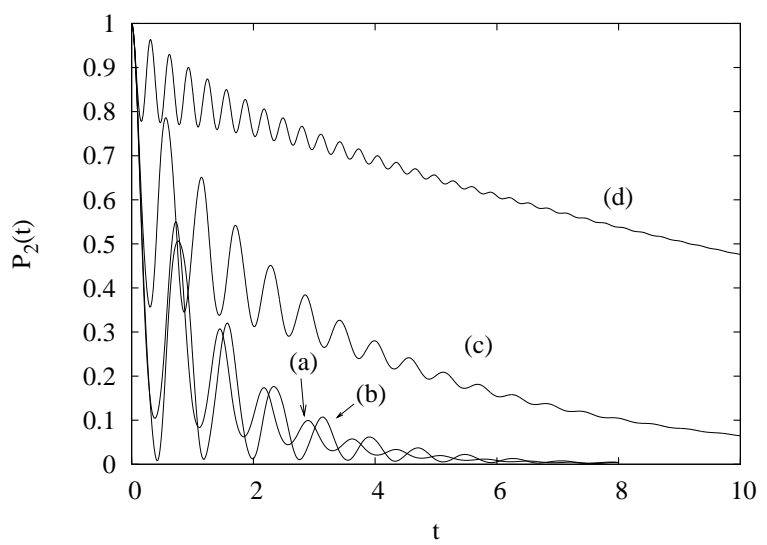

FIG. 3: High-Q cavity model. Time evolution of the probability of finding the system in state 2; $P(t)=\left|b_{2}(t)\right|^{2}$. The reservoir structure function is given by equation (32), with parameters $\Gamma=1$ and $\Omega=5.0$ in scaled units. The atomic transitions have equal frequency: $\bar{\delta}=0$. The centre of the resonance is offset from the atomic frequency by the detunings in (a)-(d) such that $\delta=0,5,10,20$. The results for (a) and (b) are computed using the integral equation method of section [ID and the results for (c) and (d) are computed using the master equation method discussed in section IV]

and 4. Figure 3] shows the case where the two atomic transitions have identical frequency and are detuned from the reservoir structure by increasing amounts [curves (a)-(d)]. It is seen that the oscillation frequency initially decreases, but then increases with the detuning. The damping of the oscillations remains about the same, but the decay of the population itself reduces as the detuning increases. For the large detuning cases $\delta=10,20$ (curves (c), (d) in figure 3) the integral equation method was very slow, and therefore these curves were computed using the master equation method to be discussed in Section IV As we will see, this method is equivalent to the integral equation method since the same reservoir structure function is involved. Figure 4 shows the situation for a fixed cavity mode detuning $\delta=4$, but with atomic transition frequencies that may be different. The chained and dotted curves in figure 4 show the effect of unequal atomic transition frequencies. In fact, since $\bar{\delta}= \pm \delta$ for these two cases, we can see from equation (11), and $\delta$ used in equation (32), that $\bar{\delta}= \pm \delta$ corresponds to the Lorentzian structure being resonant with the lower atomic transition, or the upper transition, respectively. The effect on the population dynamics is similar in both cases - the population is lost slightly less quickly, because of less efficient coupling, and the frequency of the oscillations changes. The frequency of population oscillation is reduced 


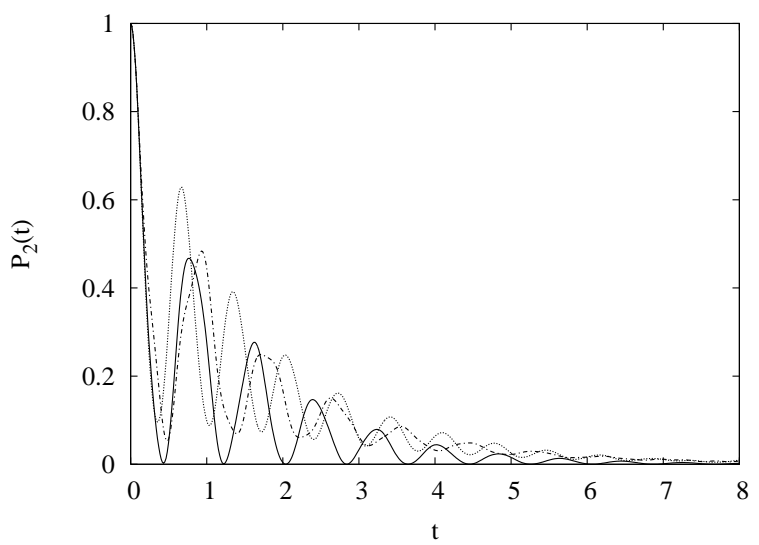

FIG. 4: High-Q cavity model. Time evolution of the probability of finding the system in state 2; $P(t)=\left|b_{2}(t)\right|^{2}$. The reservoir structure function is given by equation (32), with parameters $\Gamma=1$ and $\Omega=5.0$ in scaled units. The detuning $\delta=4$ and the off-set is $\bar{\delta}=0$ (solid line), $+\delta$ (chain), and $-\delta$ (dotted). The results are computed using the integral equation method of section ஹD.

when the structure is resonant with the lower transition, and increased when it is resonant with the upper transition. Note that for equal atomic transition frequencies both figure 3 and figure 4 display a case where the population drops close to zero periodically. This is for $\delta=5,4$ respectively.

\section{Case B: Photonic Band-Gap Model}

In this section we consider a simple model of a three-level atom coupled to a photonic band-gap system. For simplicity, we again assume equal coupling constants and transition frequencies. Thus $g_{\lambda 1}=g_{\lambda 2}=g_{\lambda}$, so that $\eta=1$. In this case the rapid frequency dependence of the reservoir structure function is due to mode frequency gaps, as a quasimode theory of such systems demonstrates [45]. A single reservoir structure function given as the difference of two Lorentzians is assumed [34] to approximately represent the frequency gap, which is assumed to be detuned from the average atomic transition frequency by $\delta$ (which is the same for both Lorentzians). In this case we let $Z_{1}=\Omega_{1}^{2}$ and $Z_{2}=-\Omega_{2}^{2}$ with $\omega_{1}^{c}=\omega_{2}^{c}=\omega^{c}$, so 
that $\delta_{1}=\delta_{2}=\delta$. Then the reservoir structure function is given by:

$$
\begin{aligned}
R_{1} & =R_{2}=R=\rho_{\lambda} g_{\lambda}^{2} \\
& =\frac{\Omega_{1}^{2}}{2 \pi} \frac{\Gamma_{1}}{\left(\omega_{\lambda}-\omega^{c}\right)^{2}+\left(\Gamma_{1} / 2\right)^{2}}-\frac{\Omega_{2}^{2}}{2 \pi} \frac{\Gamma_{2}}{\left(\omega_{\lambda}-\omega^{c}\right)^{2}+\left(\Gamma_{2} / 2\right)^{2}}, \\
& \equiv \frac{\Omega_{1}^{2}}{2 \pi} \frac{\Gamma_{1}}{(\Delta \omega-\delta)^{2}+\left(\Gamma_{1} / 2\right)^{2}}-\frac{\Omega_{2}^{2}}{2 \pi} \frac{\Gamma_{2}}{(\Delta \omega-\delta)^{2}+\left(\Gamma_{2} / 2\right)^{2}} .
\end{aligned}
$$

The Lorentzians have coupling strengths $\Omega_{1}, \Omega_{2}$ and reservoir structure widths $\Gamma_{1}$, $\Gamma_{2}$, where

$$
\begin{gathered}
\frac{\Omega_{1}^{2}}{\Gamma_{1}}=\frac{\Omega_{2}^{2}}{\Gamma_{2}} \\
\Gamma_{2}<\Gamma_{1} .
\end{gathered}
$$

These conditions are required so that $R$ is always positive and has a zero where $\Delta \omega=\delta$. This localised zero models the photonic band-gap (see [34, 35]). The atomic transition frequencies might not be equal to each other, with the difference being represented by the parameter $\bar{\delta}$, equation (11), but if $\bar{\delta}=\delta=0$ then both transition frequencies would be resonant with the zero in the reservoir structure function equation (36).

The kernel $K$ and the function $d$ are now given by (see equations (1314)):

$$
\begin{aligned}
K\left(\Delta \omega, \Delta \omega^{\prime}\right)= & \frac{1}{2 \pi} \frac{\left(\Omega_{1}^{2} \Gamma_{1}-\Omega_{2}^{2} \Gamma_{2}\right)\left(\Delta \omega^{\prime}-\delta\right)^{2}}{\left(\left(\Delta \omega^{\prime}-\delta\right)^{2}+\left(\Gamma_{1} / 2\right)^{2}\right)\left(\left(\Delta \omega^{\prime}-\delta\right)^{2}+\left(\Gamma_{2} / 2\right)^{2}\right)} \\
& \times \frac{\left(2 s+i\left(\Delta \omega+\Delta \omega^{\prime}\right)\right)}{s\left(s+i\left(\Delta \omega+\Delta \omega^{\prime}\right)\right)} \\
& \times \frac{\left(s+i(\Delta \omega+\delta)+\Gamma_{1} / 2\right)\left(s+i(\Delta \omega+\delta)+\Gamma_{2} / 2\right)}{Q_{3}(s+i(\Delta \omega+\delta))} \\
d(\Delta \omega)= & \frac{-i}{s} \frac{\left(s+i(\Delta \omega+\delta)+\Gamma_{1} / 2\right)\left(s+i(\Delta \omega+\delta)+\Gamma_{2} / 2\right)}{Q_{3}(s+i(\Delta \omega+\delta))},
\end{aligned}
$$

with

$$
Q_{3}(x)=[x+i(\bar{\delta}-\delta)]\left(x+\Gamma_{1} / 2\right)\left(x+\Gamma_{2} / 2\right)+x\left(\Omega_{1}^{2}-\Omega_{2}^{2}\right) .
$$

In equations (39)-(41) we have used relation (37).

The matrices $\mathbf{K}_{r}, \mathbf{K}_{i}$ are determined from the kernel $K$ and the matrices $\mathbf{d}_{r}, \mathbf{d}_{i}$ from an analogous equation for $d$. The amplitude $b_{2}(t)$ is obtained by numerical integration.

Typical results are shown in figure 5 for the case of two equal atomic transition frequencies, also coincident with the gap frequency. Thus $\omega_{1}=\omega_{2}=\omega^{c}$, so that $\delta=\widetilde{\delta}=0$. The parameters used are $\Gamma_{1}=4$ and $\Omega_{1}=1$ with $\Gamma_{2}=1.0,1.5$ and 2.0. Non-Markovian decay is 


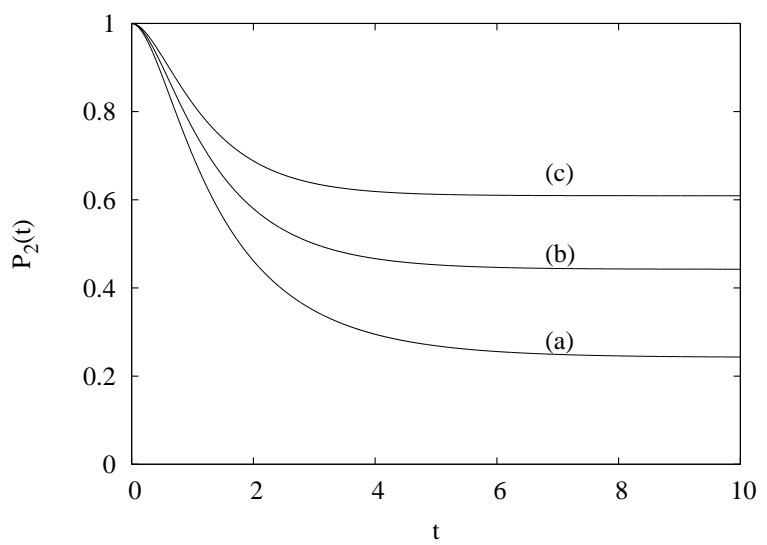

FIG. 5: Photonic band-gap model. Time evolution of the probability of finding the system in state $2 ; P(t)=\left|b_{2}(t)\right|^{2}$. The reservoir structure function is given by equation (36), with parameters $\Gamma_{1}$ $=4$ and $\Omega_{1}=1$ and: (a) $\Gamma_{2}=1$ (b) $\Gamma_{2}=1.5$ (c) $\Gamma_{2}=2.0$ in scaled units. The case of resonance is shown, where each transition is resonant with the gap: $\bar{\delta}=\delta=0$.

seen for small $t$ as the initial quadratic behaviour. Population trapping effects are found in all three cases. The amount of trapped population increases as $\Gamma_{2}$ increases.

Figure [6] shows the effect of detuning the two (equal) atom transition frequencies from the centre of the gap. The parameters used are as in figure [5] with $\Gamma_{2}=1$. Again, for the large detuning case $\delta=8$ (curve (e) in figure 6) the results were calculated using the master equation method of Section IV. We see that there is a loss of the population trapping effect, although for larger detunings $\delta$ the rate of decay is considerably reduced. However, this reduction can be attributed to the decrease in the density of states when detuned far from the gap and outside the scope of the second, positive, Lorentzian. In that sense it is an artifact of the model. The effect is eliminated for the parameters shown in figure 7 where $\Gamma_{1}$ is comparatively large. The parameters are as in figure 6 except that $\Gamma_{1}=50$ and the detunings selected are different. All the graphs in figure 6 were computed using the master equation method. In this case only the loss of the trapping is seen as the detuning increases, and the curves appear to saturate as the background density of states flattens out for the larger detunings. 


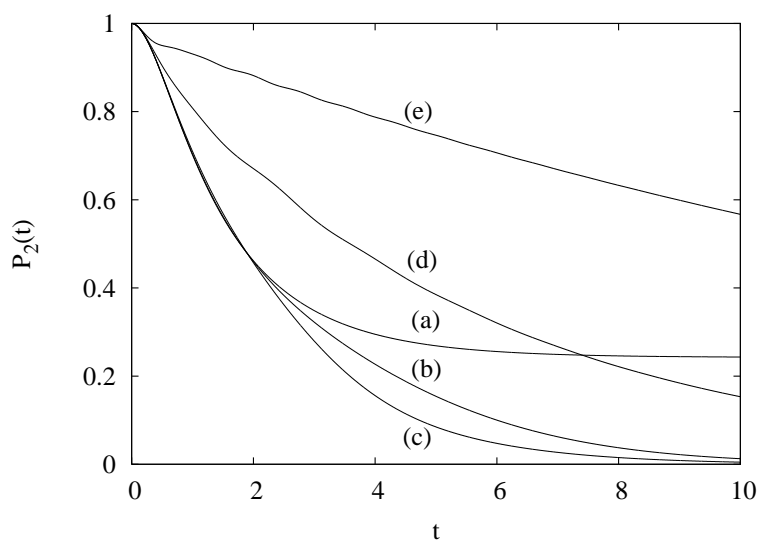

FIG. 6: Photonic band-gap model. Time evolution of the probability of finding the system in state 2; $P_{2}(t)=\left|b_{2}(t)\right|^{2}$. The reservoir structure function is given by equation (36), with parameters $\Gamma_{1}$ $=4$ and $\Omega_{1}=1$ and $\Gamma_{2}=1$ in scaled units. The atomic transitions have equal frequency: $\bar{\delta}=0$. The centre of the gap is offset from the atomic frequency by the detunings in (a)-(e) of $\delta=0,0.5,1,4$ and 8. The results for $\delta=0$ to 4 are computed using the integral equation method of section ஊD and the result for $\delta=8$ is computed using the master equation method discussed in section [V]

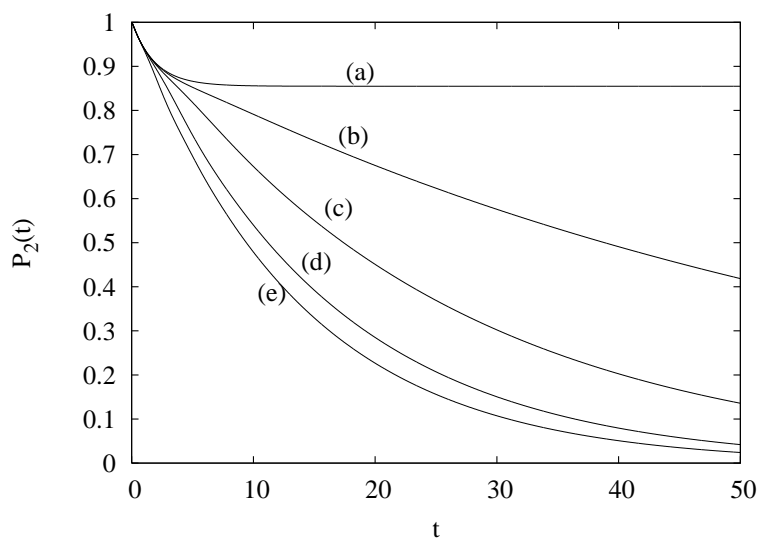

FIG. 7: Photonic band-gap model. Time evolution of the probability of finding the system in state $2 ; P_{2}(t)=\left|b_{2}(t)\right|^{2}$. The reservoir structure function is given by equation (36), with parameters $\Gamma_{1}=50$ and $\Omega_{1}=1$ and $\Gamma_{2}=1$ in scaled units. The atomic transitions have equal frequency: $\bar{\delta}=0$. The centre of the gap is offset from the atomic frequency by the detunings in (a)-(e) of $\delta=0,0.25,0.5,1$ and 2 . These results are computed using the master equation method discussed in section IV] 


\section{MASTER EQUATION THEORY}

\section{A. Pseudomode Treatment}

We have previously found that Lorentzian reservoir structures can be connected, in a nonperturbative way, to Lindblad master equations using the pseudomode approach [34, 35], which involves considering the positions and residues of the poles of the reservoir structure function in the complex frequency plane. Each pole is associated with a pseudomode.

This approach was applied to the case of a two-level atom in a high-Q cavity with a single resonance of width $\Gamma_{1}$ [34, 35]. The system takes on the dynamics of a damped Jaynes-Cummings model where the atom exchanges energy with a pseudomode, from which the energy is lost. In the case of our three-level atom in a high-Q cavity the pseudomode approach would result in a master equation of the form

$$
\frac{\mathrm{d}}{\mathrm{d} t} \hat{\rho}=-\frac{i}{\hbar}\left[\widehat{H}_{0}^{C A V}, \hat{\rho}\right]-\frac{\Gamma_{1}}{2}\left(\hat{a}_{1}^{\dagger} \hat{a}_{1} \hat{\rho}-2 \hat{a}_{1} \hat{\rho} \hat{a}_{1}^{\dagger}+\hat{\rho} \hat{a}_{1}^{\dagger} \hat{a}_{1}\right)
$$

with the Hamiltonian

$$
\widehat{H}_{0}^{C A V}=\hbar\left[\omega_{1}|1\rangle\left\langle 1\left|+\left(\omega_{1}+\omega_{2}\right)\right| 2\right\rangle\langle 2|+\omega^{c} \hat{a}_{1}^{\dagger} \hat{a}_{1}+\widehat{H}_{A F}^{(1)}\right] .
$$

Here the term $\widehat{H}_{A F}^{(1)}$ represents the atom-pseudomode coupling

$$
\widehat{H}_{A F}^{(1)}=\hbar \Omega\left(\hat{a}_{1}^{\dagger}|0\rangle\left\langle 1\left|+\hat{a}_{1}\right| 1\right\rangle\left\langle 0\left|+\hat{a}_{1}^{\dagger}\right| 1\right\rangle\left\langle 2\left|+\hat{a}_{1}\right| 2\right\rangle\langle 1|\right)
$$

Comparison of the calculated results shows that this master equation reproduces the results of Section IIIB for the atomic populations obtained by the integral equation method.

The pseudomode approach was also applied to the case of a two-level atom in a photonic band-gap system [34]. In the case of cascade atom in the band-gap material, as in Section IIIC, this approach [34, 35] would, in general, result in a two-mode master equation of the form:

$$
\begin{aligned}
\frac{\mathrm{d}}{\mathrm{d} t} \hat{\rho}= & -\frac{i}{\hbar}\left[\widehat{H}_{0}^{B G}, \hat{\rho}\right]-\frac{\Gamma_{1}^{\prime}}{2}\left(\hat{a}_{1}^{\dagger} \hat{a}_{1} \hat{\rho}-2 \hat{a}_{1} \hat{\rho} \hat{a}_{1}^{\dagger}+\hat{\rho} \hat{a}_{1}^{\dagger} \hat{a}_{1}\right) \\
& -\frac{\Gamma_{2}^{\prime}}{2}\left(\hat{a}_{2}^{\dagger} \hat{a}_{2} \hat{\rho}-2 \hat{a}_{2} \hat{\rho} \hat{a}_{2}^{\dagger}+\hat{\rho} \hat{a}_{2}^{\dagger} \hat{a}_{2}\right)
\end{aligned}
$$


with the Hamiltonian

$$
\begin{aligned}
\widehat{H}_{0}^{B G}= & \hbar\left[\omega_{1}|1\rangle\left\langle 1\left|+\left(\omega_{1}+\omega_{2}\right)\right| 2\right\rangle\langle 2|+\omega^{c} \hat{a}_{1}^{\dagger} \hat{a}_{1}+\omega^{c} \hat{a}_{2}^{\dagger} \hat{a}_{2}\right] \\
& +\hbar \frac{\sqrt{\Gamma_{1} \Gamma_{2}}}{2}\left(\hat{a}_{1}^{\dagger} \hat{a}_{2}+\hat{a}_{1} \hat{a}_{2}^{\dagger}\right) \\
& +\widehat{H}_{A F}^{(2)} .
\end{aligned}
$$

This time the atom-pseudomode coupling is to the pseudomode labelled ' 2 ', i.e.

$$
\widehat{H}_{A F}^{(2)}=\hbar \Omega_{P B G}\left(\hat{a}_{2}^{\dagger}|0\rangle\left\langle 1\left|+\hat{a}_{2}\right| 1\right\rangle\left\langle 0\left|+\hat{a}_{2}^{\dagger}\right| 1\right\rangle\left\langle 2\left|+\hat{a}_{2}\right| 2\right\rangle\langle 1|\right),
$$

similarly to equation (44). The two modes (or pseudo modes) of this case are coupled to each other in the Hamiltonian (46). For the case when the reservoir structure function has a zero, as has been prescribed by equation (37), we find that $\Gamma_{1}^{\prime}=0$. However, $\Gamma_{2}^{\prime}$ is non-zero and is related to the large $\omega$ behaviour of the reservoir structure function $R$. This parameter, and the atom-pseudomode coupling strength are given by

$$
\begin{aligned}
\Gamma_{2}^{\prime} & =\Gamma_{1}+\Gamma_{2}, \\
\Omega_{P B G} & =\sqrt{\Omega_{1}^{2}-\Omega_{2}^{2}}
\end{aligned}
$$

which are both positive. Again, comparison of the calculated results shows that the master equation (45) reproduces the results of Section IIIC for the atomic populations obtained by the integral equation method. For the full density matrix, 36 coupled differential equations are required with the two excitations produced from the three-level ladder system.

\section{B. Quasimode Treatment}

Markovian master equations describing Lorentzian reservoir structures can also be obtained based on quasimodes [43, 44], which are identified with approximate modes of the EM field. The basic concepts and equations for quasimode theory are set out in the Appendix. For the present paper we consider the case where the continuum quasimode density $\varrho_{c}(\Delta)$ and the $n$ discrete-continuum coupling constants $W_{i}(\Delta)$ are slowly varying functions of the continuum quasimode frequency $\Delta$. In this situation we obtain Markovian master equations (equation (82) ) for the density operator $\hat{\rho}$ describing the system consisting of the atom plus the $n$ discrete quasimodes. The continuum quasimodes constitute the reservoir. In addition simple expressions are obtained for the reservoir structure function $R_{k}(\omega)$ for 
the true modes [equation (84)]. These results will now be applied to the high-Q cavity and photonic band-gap cases.

For the high-Q cavity case, only a single discrete quasimode is involved. The master equation for the cascade atom plus single discrete quasimode system is then

$$
\frac{d}{d t} \hat{\rho}=\frac{-i}{\hbar}\left[\widehat{H}_{S}, \hat{\rho}\right]+\frac{\Gamma}{2}\left\{\left[\hat{a}_{1}, \hat{\rho} \hat{a}_{1}^{\dagger}\right]+\left[\hat{a}_{1} \hat{\rho}, \hat{a}_{1}^{\dagger}\right]\right\}
$$

where the decay rate $\Gamma$ is given by

$$
\Gamma=2 \pi \varrho_{c}\left|W_{1}\right|^{2}
$$

and the system Hamiltonian is

$$
\begin{aligned}
\widehat{H}_{S}= & \sum_{k=1,2} \eta_{k} \hbar \omega_{k}\left(\hat{\sigma}_{k}^{+} \widehat{\sigma}_{k}^{-}-\widehat{\sigma}_{k}^{-} \widehat{\sigma}_{k}^{+}\right)+\hbar \nu_{1} \hat{a}_{1}^{\dagger} \hat{a}_{1} \\
& +\sum_{k=1,2}\left(\hbar \lambda_{k 1}^{*} \hat{a}_{1} \widehat{\sigma}_{k}^{+}+H . c\right)
\end{aligned}
$$

with $\widehat{\sigma}_{1}^{+}=|1\rangle\left\langle 0\left|, \widehat{\sigma}_{2}^{+}=\right| 2\right\rangle\langle 1|, \eta_{1}=\frac{2}{3}+\frac{1}{3} \frac{\omega_{2}}{\omega_{1}}, \eta_{2}=\frac{2}{3}+\frac{1}{3} \frac{\omega_{1}}{\omega_{2}}$. The atomic Hamiltonian is that given in equation (2), apart from an additive constant $\left(2 \hbar \omega_{1}+\hbar \omega_{2}\right) / 3$. The choice $\lambda_{11}=$ $\lambda_{21}=\Omega$ (real) is natural since we are dealing with a situation where both transitions have equal coupling constants with the EM field, and we choose $\nu_{1}=\omega^{c}$ to have the quasimode frequency coincide with the peak in the reservoir structure function. These choices then result in the same master equation as equation (42). A straightforward evaluation of the reservoir structure function from equation (84) gives

$$
R_{k}(\omega)=\Omega^{2} \frac{\Gamma}{2 \pi} \frac{1}{\left(\omega-\omega^{c}\right)^{2}+(\Gamma / 2)^{2}}
$$

for both transitions. This result is the same as in equations (3132). Thus the high-Q cavity case can be treated via quasimode theory involving a single discrete quasimode, which may be identified as the cavity mode.

For the photonic band-gap case, two discrete quasimodes are involved. There is now more choice for the parameters in the quasimode model and it turns out we can choose these to obtain the same reservoir structure function as in equations (35 36) as well as generating a master equation which is the same as equation (45) obtained from pseudomode theory. However, to achieve this we must first introduce two new discrete quasimodes, whose 
annihilation operators $\widehat{b}_{1}, \widehat{b}_{2}$ are defined by

$$
\begin{aligned}
\widehat{b}_{1} & =\kappa^{-1 / 2} \pi \rho_{c}\left(-W_{2} \hat{a}_{1}+W_{1} \hat{a}_{2}\right) \\
\widehat{b}_{2} & =\kappa^{-1 / 2} \pi \rho_{c}\left(W_{1}^{*} \hat{a}_{1}+W_{2}^{*} \hat{a}_{2}\right) \\
\kappa & =\frac{1}{2}\left(\Gamma_{1}+\Gamma_{2}\right),
\end{aligned}
$$

where the decay rates are

$$
\begin{aligned}
& \Gamma_{1}=2 \pi \varrho_{c}\left|W_{1}\right|^{2} \\
& \Gamma_{2}=2 \pi \varrho_{c}\left|W_{2}\right|^{2} .
\end{aligned}
$$

Note that the $\widehat{b}_{1}, \widehat{b}_{2}$ and their adjoints satisfy the standard Bose commutation rules, which is a consequence of the matrix involved in (54, 55) being unitary.

From equation (82) we immediately see that the master equation is given by

$$
\frac{d}{d t} \hat{\rho}=\frac{-i}{\hbar}\left[\widehat{H}_{S}, \hat{\rho}\right]+\frac{1}{2}\left(\Gamma_{1}+\Gamma_{2}\right)\left\{\left[\widehat{b}_{2}, \hat{\rho} \widehat{b}_{2}^{\dagger}\right]+\left[\widehat{b}_{2} \hat{\rho}, \widehat{b}_{2}^{\dagger}\right]\right\}
$$

showing that relaxation only involves the new quasimode with annihilation operator $\widehat{b}_{2}$, the decay rate being the sum of the original rates $\Gamma_{1}$ and $\Gamma_{2}$. This result for the relaxation terms is the same as in the pseudomode master equation (45).

The system Hamiltonian $\widehat{H}_{S}$ can then be written in terms of the new quasimode operators by inverting equations (54, 55) to give $\hat{a}_{1}, \hat{a}_{2}$ in terms of $\widehat{b}_{1}, \widehat{b}_{2}$ and substituting in equation (83). There are still many choices that can be made for the quasimode frequencies $\nu_{i}$ $(i=1,2)$, the magnitude and phases of the atom-quasimode coupling constants $\lambda_{k i}(k=$ $1,2 ; i=1,2)$, the quasimode-quasimode coupling constant $V_{12}$ and the phase for discretecontinuum coupling constants $W_{i}(i=1,2)$. The magnitude of the $W_{i}$ are equivalent to the decay rates $\Gamma_{i}$. The atomic term in the system Hamiltonian $\widehat{H}_{S}$ is unaltered

$$
\widehat{H}_{S 1}=\sum_{k=1,2} \eta_{k} \hbar \omega_{k}\left(\widehat{\sigma}_{k}^{+} \widehat{\sigma}_{k}^{-}-\widehat{\sigma}_{k}^{-} \widehat{\sigma}_{k}^{+}\right)
$$

Since the photonic band-gap model involves two Lorentzians with the same centre frequency $\omega^{c}$ it is natural to choose the discrete quasimode frequencies $\nu_{i}$ to be both equal to $\omega^{c}$, and since both atomic transitions have equal coupling constants with the EM field it is natural to choose the atom-quasimode coupling constants to be independent of the transition. Thus

$$
\begin{aligned}
\nu_{1} & =\nu_{2}=\omega^{c} \\
\lambda_{k 1} & =\lambda_{1}, \quad \lambda_{k 2}=\lambda_{2} .
\end{aligned}
$$


We also introduce the various phase factors via the definitions

$$
\begin{aligned}
V_{12} & =\left|V_{12}\right| \exp \left(i \xi_{12}\right) \\
W_{1} & =\left|W_{1}\right| \exp \left(i \phi_{1}\right), \quad W_{2}=\left|W_{2}\right| \exp \left(i \phi_{2}\right) \\
\lambda_{1} & =\left|\lambda_{1}\right| \exp \left(i \theta_{1}\right), \quad \lambda_{2}=\left|\lambda_{2}\right| \exp \left(i \theta_{2}\right) .
\end{aligned}
$$

In view of (61) and the unitary relation between the new and original discrete quasimode operators, it follows that the quasimode energy term in $\widehat{H}_{S}$ is given by

$$
\widehat{H}_{S 2}=\hbar \omega^{c}\left(\widehat{b}_{1}^{\dagger} \widehat{b}_{1}+\widehat{b}_{2}^{\dagger} \widehat{b}_{2}\right)
$$

To obtain the required atom-quasimode interaction we choose

$$
\begin{aligned}
\left|V_{12}\right| & =\frac{1}{2} \sqrt{\Gamma_{1} \Gamma_{2}} \\
\xi_{12} & =\phi_{1}-\phi_{2}+(2 \nu+1) \frac{\pi}{2} \quad(\nu=0, \pm 1, \pm 2, . .) \\
\phi_{1}+\phi_{2} & =(2 \nu+1) \frac{\pi}{2}-\mu 2 \pi \quad(\mu=0, \pm 1, \pm 2, . .),
\end{aligned}
$$

and find that the quasimode-quasimode interaction is given by

$$
\widehat{H}_{S 3}=\hbar \frac{1}{2} \sqrt{\Gamma_{1} \Gamma_{2}}\left(\widehat{b}_{1}^{\dagger} \widehat{b}_{2}+\widehat{b}_{2}^{\dagger} \widehat{b}_{1}\right) \text {. }
$$

To obtain the required atom-quasimode coupling together with condition (62) we choose

$$
\begin{aligned}
\left|\lambda_{1}\right| & =\Omega_{1} \sqrt{\frac{\Omega_{1}^{2}-\Omega_{2}^{2}}{\Omega_{1}^{2}+\Omega_{2}^{2}}}, \quad\left|\lambda_{2}\right|=\Omega_{2} \sqrt{\frac{\Omega_{1}^{2}-\Omega_{2}^{2}}{\Omega_{1}^{2}+\Omega_{2}^{2}}} \\
\theta_{1}-\phi_{1} & =\theta_{2}-\phi_{2} \\
& =\xi 2 \pi \quad(\xi=0, \pm 1, \pm 2, . .),
\end{aligned}
$$

where the (real) quantities $\Omega_{1}, \Omega_{2}, \Gamma_{1}$ and $\Gamma_{2}$ are related as in equations (37] 38). We thus find that

$$
\widehat{H}_{S 4}=\hbar \Omega_{P B G}\left(\widehat{b}_{2}\left(\widehat{\sigma}_{1}^{+}+\widehat{\sigma}_{2}^{+}\right)+\widehat{b}_{2}^{\dagger}\left(\widehat{\sigma}_{1}^{-}+\widehat{\sigma}_{2}^{-}\right)\right)
$$

where, as in equation (49),

$$
\Omega_{P B G}=\sqrt{\Omega_{1}^{2}-\Omega_{2}^{2}}
$$

Overall, with $\widehat{H}_{S}=\widehat{H}_{S 1}+\widehat{H}_{S 2}+\widehat{H}_{S 3}+\widehat{H}_{S 4}$ the system Hamiltonian (and hence the master equation) is the same as that obtained via the pseudomode approach. The key feature is that we started with two original coupled discrete quasimodes with equal frequencies, whose 
parameters satisfy the conditions in equations (61, 38, 67, 68, 69, 71, 172, 73). Two new discrete quasimodes were introduced, also coupled and with equal frequencies. However, the first of these new quasimodes $\widehat{b}_{1}$ is neither coupled to the atomic transitions nor involved in relaxation. The other new quasimode $\widehat{b}_{2}$ is coupled to the atomic transitions and is involved in relaxation with a rate equal to the sum of the original rates.

Finally, with the conditions referred to in the last paragraph applying, it is a straightforward process to evaluate the reservoir structure function from equation (84). We find that this is the same for both transitions and is given by

$$
\begin{aligned}
R_{k}(\omega)= & R(\omega) \\
= & \Omega_{1}^{2} \frac{\Gamma_{1}}{2 \pi} \frac{1}{\left(\omega-\omega^{c}\right)^{2}+\left(\Gamma_{1} / 2\right)^{2}} \\
& -\Omega_{2}^{2} \frac{\Gamma_{2}}{2 \pi} \frac{1}{\left(\omega-\omega^{c}\right)^{2}+\left(\Gamma_{2} / 2\right)^{2}} .
\end{aligned}
$$

Thus the photonic band-gap case can be treated via quasimode theory involving two coupled discrete quasimodes of equal frequency, where one may be identified as a band-gap mode and the other as a background mode.

\section{SUMMARY}

The dynamical behaviour of a three level cascade atom coupled to a structured reservoir (typically of EM field modes), and initially in the upper state has been analysed via Laplace transform and exact master equation methods. In the Laplace transform approach, the atomic density operator is determined from the solutions of integral equations, in which the properties of the structured reservoir only appears via reservoir structure functions, all essentially given by the product of the mode density times the square of coupling constants. In the cascade system two distinct reservoir structure functions are involved. The dependence of the dynamics solely on reservoir structure functions is required for treating structured reservoir problems via pseudomode theory, so our results suggest that it may be possible to

extend pseudomode theory to problems involving more than a single photon excitation of the reservoir.

Solutions of the integral equation involve discretising the frequency space into $N$ points, so the matrix inversion step involves a matrix with $4 N^{2}$ elements compared to, say, $\mathcal{O}\left(N^{4}\right)$ elements represented by the original coupled amplitude equations in an equivalent discretised 
form. Considerations based on analytic continuation were required for obtaining solutions of the problem using numerical methods, including carrying out an inverse Laplace transform. For some oscillatory problems (specifically, with large detunings) there were numerical difficulties with the integral equation approach.

Two important physical situations have been studied using these numerical methods. The first applies to a simple model of a three-level atom with identical cascade transitions coupled to a single high-Q cavity mode, involving a single Lorentzian reservoir structure function. Non-Markovian oscillatory decay of the excited state has been demonstrated. The second applies to a model of a three-level atom with identical cascade transitions coupled to a photonic band-gap system, involving a single reservoir structure function modelled simply as the difference between two Lorentzian functions. Again we have shown the non-Markovian oscillatory decay of the excited state and population trapping effects.

We have also studied both the high-Q cavity and the photonic band-gap cases via the master equation method. The master equation can be obtained both from the pseudomode approach and the quasimode approach, and numerical calculations of the upper state probability via the master equation method give the same results as the integral equation method based on essential states. This shows that complicated non-Markovian decays into structured EM field reservoirs can be described by Markovian models in which the atomic system is augmented by one or two pseudomodes or quasimodes, which in the quasimode approach themselves undergo Markovian relaxation into a flat reservoir. For the high-Q cavity case the single pseudomode or quasimode may be identified as the cavity mode, for the photonic band-gap case the two coupled pseudomodes or quasimodes may be identified as band-gap and background modes.

\section{ACKNOWLEDGEMENTS}

This paper is dedicated to the memory of Edwin Power, whose pioneering work on Quantum Electrodynamics applied to atomic and molecular systems was central to the development of Quantum Optics. Helpful discussions with R. Blatt, A. Caldeira, H. Carmichael, M. Collett and C. Savage are gratefully acknowledged. 


\section{APPENDIX : QUASIMODE THEORY}

In quasimode theory [43, 44] an atomic system with upward, downward transition operators $\widehat{\sigma}_{k}^{+}, \widehat{\sigma}_{k}^{-}$and transition frequencies $\omega_{k}$ is coupled to a set of $n$ discrete bosonic quasimodes with annihilation, creation operators $\hat{a}_{i}, \hat{a}_{i}^{\dagger}$ and frequencies $\nu_{i}$. The coupling constants are denoted $\lambda_{k i}$. The discrete quasimodes may be coupled to each other with coupling constants $V_{i j}$. In addition, there is a coupling between the discrete quasimodes and a continuum of quasimodes whose annihilation, creation operators are $\widehat{b}(\Delta), \widehat{b}(\Delta)^{\dagger}$ and whose frequencies are $\Delta$. All coupling terms are in the RWA. The continuum quasimodes have mode density $\varrho_{c}(\Delta)$ and the coupling constant with the discrete quasimodes are denoted $W_{i}(\Delta)$. The continuum quasimodes are neither coupled to each other nor to the atomic system. In Refs. 43, 44] the quasimodes were taken to be approximations to the true EM field modes. The overall Hamiltonian is given by

$$
\widehat{H}=\widehat{H}_{A}+\widehat{H}_{Q}+\widehat{H}_{A Q}
$$

with

$$
\begin{aligned}
\widehat{H}_{A}= & \sum_{k} \eta_{k} \hbar \omega_{k}\left(\widehat{\sigma}_{k}^{+} \widehat{\sigma}_{k}^{-}-\widehat{\sigma}_{k}^{-} \widehat{\sigma}_{k}^{+}\right) \\
\widehat{H}_{Q}= & \sum_{i} \hbar \nu_{i} \hat{a}_{i}^{\dagger} \hat{a}_{i}+\sum_{i \neq j} \hbar V_{i j} \hat{a}_{i}^{\dagger} \hat{a}_{j} \\
& +\sum_{i} \int d \Delta \varrho_{c}(\Delta)\left[\hbar W_{i}(\Delta) \hat{a}_{i}^{\dagger} \widehat{b}(\Delta)+H . c .\right] \\
& +\int d \Delta \varrho_{c}(\Delta) \hbar \Delta \widehat{b}(\Delta)^{\dagger} \widehat{b}(\Delta) \\
\widehat{H}_{A Q}= & \sum_{k} \sum_{i}\left(\hbar \lambda_{k i}^{*} \hat{a}_{i} \widehat{\sigma}_{k}^{+}+H . c\right) .
\end{aligned}
$$

In equation (78) the quantities $\eta_{k}$ are chosen so that $\widehat{H}_{A}$ equals the atomic Hamiltonian given in equation (2), apart from an additive constant $\left(2 \hbar \omega_{1}+\hbar \omega_{2}\right) / 3$. The annihilation and creation operators for the discrete quasimodes satisfy the Kronecker delta commutation rules, whilst those for the continuum quasimodes satisfy Dirac delta function commutation rules:

$$
\begin{aligned}
{\left[\hat{a}_{i}, \hat{a}_{j}^{\dagger}\right] } & =\delta_{i j} \\
{\left[\widehat{b}(\Delta), \widehat{b}\left(\Delta^{\prime}\right)^{\dagger}\right] } & =\delta\left(\Delta-\Delta^{\prime}\right) / \varrho_{c}(\Delta) .
\end{aligned}
$$

The $\varrho_{c}$ factor on the right side of the last equation gives annihilation and creation operators that are dimensionless. 
In Refs. [43, 44] the true EM field modes were described by annihilation and creation operators $\widehat{A}(\omega)$ and $\widehat{A}(\omega)^{\dagger}$ that were obtained by Fano diagonalisation methods. This involved writing $\widehat{A}(\omega)$ as a linear combination of the discrete quasimode annihilation operators $\hat{a}_{i}$ plus an integral of the continuum annihilation operators $\widehat{b}(\Delta)$, and requiring that the true mode annihilation operator is an eigenoperator for the quasimode Hamiltonian (79) with energy $\hbar \omega$. Expressions for the atom-quasimode coupling (80) could then be written in terms of the true mode annihilation and creation operators (equations (18), (19) of Ref. [44]) and a result for the reservoir structure function $R_{k}(\omega)$ associated with the $k$ atomic transition obtained (equation (50) of Ref. [44]). These results applied to the general case where the continuum quasimode density $\varrho_{c}(\Delta)$ and the discrete-continuum coupling constants $W_{i}(\Delta)$ were not necessarily slowly varying functions of $\Delta$.

For the present paper we are interested in the situation where the dynamics of the system consisting of the atom plus the $n$ discrete quasimodes is Markovian. The reservoir consists of the continuum quasimodes. Markovian behaviour occurs when $\varrho_{c}(\Delta)$ and the $W_{i}(\Delta)$ are slowly varying functions of $\Delta$ and can be treated as constant. The flatness of the continuum quasimode density and the coupling constants results in the reservoir correlation time being short enough for Markovian behaviour to occur. The density operator $\hat{\rho}$ for the system consisting of the atom plus the $n$ discrete quasimodes can then be shown to satisfy the Markovian master equation

$$
\frac{d}{d t} \hat{\rho}=\frac{-i}{\hbar}\left[\widehat{H}_{S}, \hat{\rho}\right]+\sum_{i j} \pi \varrho_{c} W_{i} W_{j}^{*}\left\{\left[\hat{a}_{j}, \hat{\rho} \hat{a}_{i}^{\dagger}\right]+\left[\hat{a}_{j} \hat{\rho}, \hat{a}_{i}^{\dagger}\right]\right\},
$$

where the system Hamiltonian is

$$
\begin{aligned}
\widehat{H}_{S}= & \sum_{k} \eta_{k} \hbar \omega_{k}\left(\widehat{\sigma}_{k}^{+} \widehat{\sigma}_{k}^{-}-\widehat{\sigma}_{k}^{-} \widehat{\sigma}_{k}^{+}\right)+\sum_{i} \hbar \nu_{i} \hat{a}_{i}^{\dagger} \hat{a}_{i} \\
& +\sum_{i \neq j} \hbar V_{i j} \hat{a}_{i}^{\dagger} \hat{a}_{j}+\sum_{k} \sum_{i}\left(\hbar \lambda_{k i}^{*} \hat{a}_{i} \hat{\sigma}_{k}^{+}+H . c\right) .
\end{aligned}
$$

In this case of a flat continuum the reservoir structure function is given by

$$
R_{k}(\omega)=\varrho_{c} \frac{\left|Q_{n-1}^{k}(\omega)\right|^{2}}{\left|P_{n}(\omega)\right|^{2}}
$$

where

$$
Q_{n-1}^{k}(\omega)=\sum_{i j} \lambda_{k i}\left(\omega \mathbf{E}_{n}-\Omega\right)_{i j}^{A D J} W_{j}^{*}
$$




$$
P_{n}(\omega)=\left|\omega \mathbf{E}_{n}-\Omega\right|-i \pi \varrho_{c} \sum_{i j} W_{i}\left(\omega \mathbf{E}_{n}-\Omega\right)_{i j}^{A D J} W_{j}^{*}
$$

and the $n \times n$ matrix $\Omega$ has elements

$$
\Omega_{i j}=\nu_{i} \delta_{i j}+\left(1-\delta_{i j}\right) V_{j i}
$$

with the adjugate matrix $\left(\omega \mathbf{E}_{n}-\Omega\right)^{A D J}$ related to the inverse via

$$
\left(\omega \mathbf{E}_{n}-\Omega\right)^{-1}=\frac{\left(\omega \mathbf{E}_{n}-\Omega\right)^{A D J}}{\left|\omega \mathbf{E}_{n}-\Omega\right|}
$$

In obtaining this result we note that the frequency shift matrix $F_{i j}(\omega)$ (equation (27) of Ref. [44]) is zero.

[1] M. O. Scully and M. S. Zubairy, Quantum Optics, Cambridge University Press, (Cambridge, 1997)

[2] G. Auletta, Foundations and Interpretation of Quantum Mechanics, World Scientific, (Singapore, 2001)

[3] W. H. Zurek, Rev. Mod. Phys. 75, 715 (2003)

[4] W. H. Zurek, S. Habib and J. P. Paz, Phys. Rev. Lett. 70, 1187 (1993)

[5] L. Pitaevskii and S. Stringari, Bose-Einstein Condensation, Clarendon Press, (Oxford, 2003)

[6] S. M. Barnett and P. M. Radmore, Methods in Theoretical Quantum Optics, (Oxford University Press, Oxford 1997)

[7] J. J. Hope, G. M. Moy, M. J. Collett and C. M. Savage, Phys. Rev. A 61, 023603 (2000)

[8] W. T. Strunz and T. Yu, Phys. Rev. A 69, 052115 (2004)

[9] H-P. Breuer and B. Kappler, Ann.Phys. (NY) 291, 36 (2001)

[10] P. Warszawski and H. M. Wiseman, J. Opt. B: Quantum Semiclass. Opt. 5, 1 (2003)

[11] V. Privman and D. Tolkunov, e-print cond-matt/0405694

[12] D. P. DiVincenzo and D. Loss, Phys. Rev. B 71, 035318 (2005)

[13] B. J. Dalton, J. Mod. Opt. 50, 951 (2003)

[14] D. Braun, F. Haake and W. T. Strunz, Phys. Rev. Lett. 86, 2913 (2001)

[15] W. T. Strunz, F. Haake and D. Braun, Phys. Rev. A 67, 022101 (2003)

[16] W. T. Strunz and F. Haake, Phys. Rev. A 67, 022102 (2003) 
[17] P. Lambropoulos, G. M. Nikolopoulos, T. R. Nielsen and S. Bay, Rep. Prog. Phys. 63, 455 (2000)

[18] R. Zwanzig, Physica (Amsterdam) 30, 1109 (1964)

[19] S. Nakajima, Prog. Theor. Phys. 20, 948 (1958)

[20] B. J. Dalton, J. Phys. A 15, 2157 (1982)

[21] S. M. Barnett and S. Stenholm, Phys. Rev. A 64, 033808 (2001)

[22] F. Shibata, Y. Takahashi and H. Hashitsuma, J. Stat. Phys. 17, 171 (1977)

[23] J. C. Cresser, Laser Phys. 10, 1 (2000)

[24] H-P. Breuer, B. Kappler and F. Petruccione, Phys. Rev. A 59, 1633 (1999)

[25] W. T. Strunz, L. Diosi and N. Gisin, Phys. Rev. Lett. 82, 1801 (1999)

[26] M. W. Jack, M. J. Collett and D. F. Walls, Phys. Rev. A 59, 2306 (1999)

[27] K. Mølmer and S. Bay, Phys. Rev. A 59, 904 (1999)

[28] T. Quang and S. John, Phys. Rev. A 56, 4273 (1997)

[29] P. Stenius and A. Imamoḡlu, Quantum Semiclass. Opt. 8, 283 (1996)

[30] H-P. Breuer, Phys. Rev. A 70, 01206 (2004)

[31] E. Paspalakis, D. Angelakis and P. L. Knight, Opt. Comm. 172, 229 (1999)

[32] S. Bay, P. Lambropoulos and K. Mølmer, Phys. Rev. Lett. 79, 2654 (1997)

[33] C. K. Law, T. W. Chen and P. T. Leung, Phys. Rev. A 61, 023808 (2000)

[34] B. M. Garraway, Phys. Rev. A 55, 2290 (1997)

[35] B. M. Garraway, Phys. Rev. A 55, 4636 (1997)

[36] U. Fano, Phys. Rev. 124, 1866 (1961)

[37] J. Jeffers, P. Horak, S. M. Barnett and P. M. Radmore, Phys. Rev. A 62, 043602 (2000)

[38] V. Privman, Mod. Phys. Lett. B 16, 459 (2002)

[39] V. Privman, J. Stat. Phys. 110, 957 (2003)

[40] D. Tolkunov and V. Privman, Phys. Rev. A 69, 062309 (2004)

[41] L-M. Duan and G-C. Guo, Phys. Rev. A 56, 4466 (1997)

[42] J. C. Retamel and N. Zagury, Phys. Rev. A 63, 032106 (2001)

[43] B. J. Dalton, S. M. Barnett and B. M. Garraway, Fortschr. Phys. 49, 927 (2001)

[44] B. J. Dalton, S. M. Barnett and B. M. Garraway, Phys. Rev. A 64, 053813 (2001)

[45] B. J. Dalton and B. M. Garraway, J. Mod. Opt. 49, 947 (2002)

[46] B. J. Dalton and B M. Garraway, Phys. Rev. A 68, 033809 (2003) 
[47] G. M. Nikolopoulos and P. Lambropoulos, Phys. Rev. A 61, 053812 (2000)

[48] S. Bay and P. Lambropoulos, Opt. Comm. 146, 130 (1998) 